\title{
EVALUACIÓN DE ENLACES INALÁMBRICOS URBANOS USANDO PROTOCOLO IEEE 802.11b
}

\author{
Cristian Correa A. ${ }^{1} \quad$ Rodrigo Godoy S. $^{1} \quad$ Walter Grote H. $^{1} \quad$ Manuel Orellana F. $^{1}$ \\ Recibido el 20 de junio de 2005, aceptado el 10 de octubre de 2005
}

\begin{abstract}
RESUMEN
En el presente trabajo se propone un modelo de diseño de enlaces fijos en la banda de $2.45 \mathrm{GHz}$ para aplicaciones de dispositivos WiFi en ambientes urbanos. Este modelo, que está basado en el de Okumura-Hata, permite predecir el nivel de pérdidas en una transmisión inalámbrica de acuerdo al grado de obstrucción existente entre el transmisor y receptor basado en una inspección visual. El modelo se contrasta con medidas experimentales obteniéndose una predicción cuyo error no supera un margen de $5 \mathrm{~dB}$ en el $90 \%$ de los casos. Además, se establece el tiempo de coherencia de estos enlaces mediante medidas experimentales de largo plazo de la señal recibida.
\end{abstract}

Palabras clave: Modelo de predicción de pérdidas de enlace, protocolo IEEE 802.11b, tiempo de coherencia de canal.

\section{ABSTRACT}

In this publication a link loss prediction model for fixed outdoor urban wireless WiFi communications using the $2.45 \mathrm{GHz}$ frequency band based on the Okumura-Hata model is proposed. The model evaluates the expected path losses using a modified Okumura's prediction model and eye inspection of the link to determine the degree of obstruction. Results are checked against experimental data to find that in $90 \%$ of the studied links it is accurate within a $5 \mathrm{~dB}$ margin. We also analyze the coherence time of these links by performing long term measurements of the received signal.

Keywords: Link loss prediction model, IEEE 802.11 b protocol, channel coherence time.

\section{INTRODUCCIÓN}

El desarrollo de aplicaciones basadas en las telecomunicaciones inalámbricas ha sido considerable. Gran parte de estas aplicaciones apuntan a proveer un servicio inalámbrico a terminales móviles, aunque algunos enlaces son establecidos con terminales en ubicaciones fijas. En el diseño de los enlaces fijos es deseable obtener resultados cercanos a la realidad.

La señal recibida en los sistemas inalámbricos es afectada por las pérdidas de espacio libre, por multitrayectorias y por las obstrucciones existentes entre transmisor y receptor. En el proceso de diseño de los enlaces se procura poder predecir su efecto. Los modelos conocidos en la literatura son aquellos basados en un estudio elaborado que considera un levantamiento de perfil, aplicando modelos tales como propagación en espacio libre, modelo de dos rayos, obstrucción y la aplicación de los anillos de Fresnel, Longley-Rice y Durkin, todos los cuales son muy buenos para la evaluación de enlaces fijos, pero que requieren de recursos de tiempo, conocimiento e infraestructura considerables [2]. Otros modelos, que se aplican a comunicaciones móviles son Okumura, Okumura-Hata, Walfisch-Bertoni, los que permiten una predicción razonable con un grado de complejidad de cálculo que van de lo simple a lo más complejo, basados en medidas experimentales o características físicas de los enlaces [2]. Sin embargo, sus resultados son demasiado pesimistas cuando se aplican a enlaces fijos, como se demuestra en este trabajo. Es conveniente entonces considerar la posibilidad de plantear un modelo que tenga la simplicidad que presenta el modelo de predicción de Okumura, acompañado con una inspección visual del enlace para garantizar una determinada calidad con un error de predicción bajo. En este trabajo se evalúa la posibilidad de establecer un modelo de predicción de enlaces fijos confiable, contrastando los resultados obtenidos mediante éste con enlaces de largo alcance para aplicaciones WLL utilizando protocolo IEEE $802.11 \mathrm{~b}$.

1 Universidad Técnica Federico Santa María, Av. España 1680, Valparaíso, Chile, wgh@elo.utfsm.cl 


\section{MODELOS}

Es sabido que al propagarse una señal electromagnética por el ambiente sufre pérdidas, que deben ser establecidas en la fase de diseño para determinar si el nivel de recepción es suficiente para mantener un enlace con una calidad de servicio razonable. A continuación se hará un análisis resumido de los modelos de predicción existentes, para luego introducir el modelo propuesto por los autores.

Modelo de propagación en espacio libre [2]: Mediante este modelo podemos obtener las pérdidas (PL: path loss) por propagación en espacio libre presente en cada uno de los enlaces a realizar y cuando el receptor está a una distancia " $d$ ' del transmisor. Lo anterior es representado por la ecuación (1).

$$
P L(d B)=-10 \log \left(\frac{G_{t} G_{r} \lambda^{2}}{(4 \pi)^{2} d^{2}}\right)
$$

donde:

$G_{t}:$ Ganancia de la antena transmisora.

$G_{r}$ : Ganancia de la antena receptora.

$\lambda^{\prime}$ : Longitud de onda en metros.

$d$ : Distancia entre la antena transmisora y receptora en $[\mathrm{m}]$.

Modelo de Okumura, [2]: El modelo de Okumura es utilizado para predecir la potencia en un receptor ubicado en un área urbana para comunicaciones móviles. Este modelo es aplicable para el rango de frecuencias entre 150 a $1920 \mathrm{MHz}$, pero puede ser extrapolado sobre los $3.000 \mathrm{MHz}$. Según este modelo, la distancia máxima de separación que puede existir entre el transmisor y el receptor es de hasta $100 \mathrm{~km}$. Las pérdidas existentes en el enlace pueden ser obtenidas mediante la ecuación (2).

$$
L_{50}(d B)_{\text {Okumura }}=P L+A_{m u}(f, d)-G\left(h_{t e}\right)-G\left(h_{r e}\right)-G_{\text {Area }}
$$

donde:

$L_{50}(d B)$ : Pérdidas por propagación al $50 \%$ de recepción de señal.

$A_{m u}(f, d)$ : Atenuación media relativa al espacio libre.

$G\left(h_{t d}\right)$ : Ganancia de la antena transmisora [dB].

$G\left(h_{r d}\right) \quad$ : Ganancia de la antena receptora [dB].

$G_{\text {Area }} \quad$ : Factor de corrección dependiente del entorno del lugar.

Para especificar la ganancia debido a la posición de la antena receptora y transmisora y la atenuación media se recomienda al lector leer [2] y [6]. El factor de corrección que se aplicará a los enlaces analizados en este trabajo es el suburbano, debido al tipo de edificación que se encuentra en Valparaíso. Corresponde a $13 \mathrm{~dB}$ a 2.450 $\mathrm{MHz}[2]$.

Modelo Hata, [2]: El modelo Hata formula empíricamente los gráficos de pérdidas por propagación del modelo Okumura. Las pérdidas existentes en el enlace pueden ser obtenidas por medio de la ecuación (3), en que se ha incluido el factor de corrección suburbano [2].

$$
\begin{aligned}
L_{50}(d B)_{\text {Hata }} & =69.12+26.16 \cdot \log \left(f_{c}\right)-13.82 \cdot \log \left(h_{\text {te }}\right)+ \\
& -3.2\left(\log 11.75 h_{r e}\right)^{2}+ \\
& +\left(44.9-6.55 \cdot \log \left(h_{t e}\right)\right) \cdot \log (d)-2\left(\log \left(\frac{f_{c}}{28}\right)\right)^{2}
\end{aligned}
$$

donde:

$L_{50}(d B)$ : Pérdidas por propagación al $50 \%$ de recepción de señal.

$f_{c} \quad:$ Frecuencia portadora en MHz.

$h_{t e} \quad:$ Altura efectiva de la antena transmisora en metros.

$h_{t e} \quad$ : Altura efectiva de la antena receptora en metros.

$d \quad$ : Distancia entre el transmisor y el receptor en $\mathrm{km}$.

Modelo Hata-Extendido: También conocido como modelo COST-231, esta extensión del modelo Hata puede ser utilizado para un rango de frecuencia entre $1.500 \mathrm{y}$ $2.000 \mathrm{MHz}$, pero puede ser extrapolado y ser aplicado a los $2.45 \mathrm{GHz}$. La altura efectiva del transmisor va desde 30 a $200 \mathrm{~m}$, la altura efectiva del receptor desde 1 a $10 \mathrm{~m}$ y la distancia entre el transmisor y receptor debe estar en el rango 1 a $20 \mathrm{~km}$. Las pérdidas de enlace pueden ser obtenidas mediante la ecuación (4).

$$
\begin{aligned}
L_{50}(d B)_{\text {Hata-Ext }} & =51.27+33.9 \log \left(f_{c}\right)-13.82 \log \left(h_{t e}\right) \\
& -3.2\left(\log 11.75 h_{r e}\right)^{2}+\left(44.9-6.55 \log \left(h_{t e}\right)\right) \log (d)
\end{aligned}
$$

La simbología de los términos y los supuestos que se han aplicado a esta ecuación son idénticos al modelo Hata.

\section{MODELO DE ENLACES URBANOS FIJOS}

El Modelo de Enlaces Urbanos Fijos (MEnUF) propuesto en este trabajo pondera las pérdidas producidas por el efecto de propagación en espacio libre y las pérdidas adicionales propuestas por el modelo Okumura, Hata o Hata-Extendido, dado el hecho de que los enlaces son 
fijos. Para el análisis de este modelo se identifican cuatro categorías de obstrucciones de la primera zona de Fresnel del enlace, basados en la estimación que realizará el instalador cuando desee realizar la factibilidad del enlace.

Las zonas de Fresnel son regiones sucesivas del espacio, donde el trayecto de un rayo imaginario entre transmisor y receptor presenta un largo eléctrico que se encuentra entre (n-1) $\lambda / 2$ y $n \lambda / 2$ veces respecto del haz directo, donde $\mathrm{n}$ es un número natural. La figura 1 muestra un plano virtual localizado entre el transmisor y receptor. Los círculos concéntricos en el plano forman el lugar geométrico del origen de todos los haces que se propagan hacia el receptor, que cumplen con la condición que el trayecto total recorrido aumenta $\lambda / 2$ entre círculos sucesivos. Los círculos son llamados zonas de Fresnel, y las contribuciones más relevantes de la señal que se obtiene en el receptor son las de las primeras zonas de Fresnel, es decir, aquellas para las cuales $n=1,2,3$, 4 [2].

Los criterios de MEnUF se definen seguidamente:

Obstrucción Leve (L): Enlaces donde las obstrucciones se encuentran a un nivel muy inferior respecto de la línea directa de visión entre transmisor y receptor $(90 \%$ de la primera zona de Fresnel despejada). La propagación puede ser considerada equivalente a espacio libre [1].

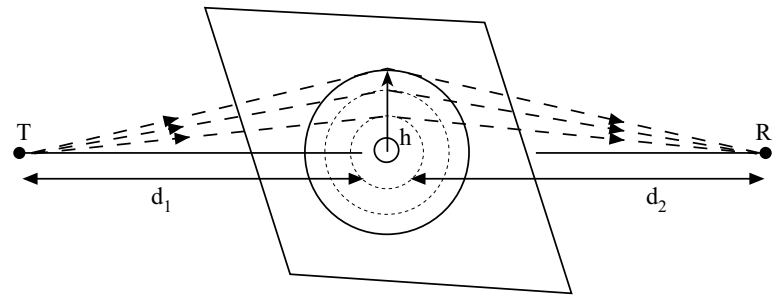

Fig. 1 Zonas de Fresnel.

Obstrucción Parcial (P): Enlaces donde las obstrucciones se encuentran a un nivel cercano a la línea de visión entre las antenas transmisoras y receptoras. En esta categoría es posible asegurar que existe, al menos, un $70 \%$ de la primera zona de Fresnel sin obstrucción [1].

Obstrucción Critica $(C)$ : Enlaces donde las obstrucciones se encuentran a un mismo nivel que la línea directa de visión entre la antena transmisora y receptora, por lo que es posible asegurar que la mitad de la primera zona de Fresnel se encuentra despejada [1].

Obstrucción Grave $(G)$ : Enlaces donde las obstrucciones se encuentran a un nivel por sobre la línea directa de visión entre la antena transmisora y receptora, por lo que la primera zona de Fresnel se encuentra despejada en menos del $30 \%$.
A continuación, se define el coeficiente de Okumura (CO), ecuación (5), el de Hata $(\mathrm{CH}),(6)$ y HataExtendido (CHE), (7), con el fin de ponderar el nivel de obstrucción que sufre el enlace en el cálculo de las pérdidas por propagación.

$$
\begin{gathered}
C O(d B)=A_{m u}(f, d)-G\left(h_{t e}\right)-G\left(h_{r e}\right)-G_{\text {Area }} \\
C H(d B)=L_{50}(d B)_{\text {Hata }}-P L \\
C H E(d B)=L_{50}(d B)_{\text {Hata }-E x t}-P L
\end{gathered}
$$

La atenuación de los radioenlaces se calcula aplicando el criterio de ponderación MEnUF al modelo de Okumura, Hata y Hata-Extendido, de acuerdo al nivel de obstrucción registrado y se define en las ecuaciones (8), (9) y (10), respectivamente.

$$
\begin{gathered}
L_{\text {TROkumura }}=P L+(\% C O) \cdot C O(d B) \\
L_{\text {TR Hata }}=P L+(\% C H) \cdot C H(d B) \\
L_{\text {TR Hata }-E x t}=P L+(\% C H E) \cdot C H E
\end{gathered}
$$

Tabla 1 Ponderación los coeficientes de acuerdo al nivel de obstrucción presente.

\begin{tabular}{|l|c|}
\hline \multicolumn{1}{|c|}{ MEnUF } & $\begin{array}{c}\text { \%CO, \%CH } \\
\mathbf{y} \% \mathbf{C H E}\end{array}$ \\
\hline Obstrucción Leve (L) & 10 \\
\hline Obstrucción Parcial (P) & 30 \\
\hline Obstrucción Critica (C) & 60 \\
\hline Obstrucción Grave (G) & 90 \\
\hline
\end{tabular}

Finalmente, la potencia en el receptor queda expresada según el resultado del MEnUF y (11).

$$
P_{R X}=P_{T X}+\left.\sum G \& P\right|_{T X}+\left.\sum G \& P\right|_{R X}-L_{T R}
$$

donde:

$P_{R X} \quad$ : Potencia recibida en el receptor.

$P_{T X} \quad$ : Potencia de transmisión.

$G \quad:$ Ganancias en transmisor y receptor.

$P \quad$ : Pérdidas en transmisor y receptor.

$L_{T R} \quad$ : Atenuación de los radioenlaces obtenida por MEnUF.

\section{ANÁLISIS EXPERIMENTAL}

Para implementar y comprobar el MEnUF, fueron instalados un equipo AP100 ORiNOCO operando en modo de infraestructura básica COR en la Universidad Técnica Federico Santa María (RED-U) con potencia de 
transmisión de $15 \mathrm{dBm}$ y dos retransmisores de igual modelo y marca operando en modo de infraestructura básica ROR en los cerros Barón (RED-M) y Playa Ancha (RED-L) de la ciudad de Valparaíso, cada uno con una potencia de transmisión de $27 \mathrm{dBm}$. De esta forma fue posible implementar enlaces en varios puntos de la ciudad con distintos escenarios y niveles de obstrucción. El equipo receptor utilizado en este trabajo tiene una antena de $24 \mathrm{dBi}$ de ganancia conectada a una tarjeta inalámbrica montada sobre un computador portátil, cuya sensibilidad de recepción es de $-80 \mathrm{dBm} @$ BER $10^{-5}$. Además, las muestras fueron tomadas cada $100 \mathrm{~ms}$ y el tiempo total de las mediciones fue de aproximadamente 1 hora.

Procedimiento: A continuación, se presenta el procedimiento seguido para la obtención de los datos y su posterior análisis:

1. Seleccionar el lugar de medición.

2. Clasificar el lugar según el grado de obstrucción presente (leve, parcial, critico o grave).

3. Obtener la distancia existente entre el equipo transmisor y receptor usando los datos de posicionamiento proporcionados por un GPS.

4. Implementar, configurar y poner en servicio el enlace.

5. Adquirir los datos mediante algún software de adquisición. En este trabajo fueron utilizados los software llamados Network Stumbler y Client Manager (Orinoco).

6. Predecir de manera teórica, mediante el MEnUF el nivel de señal esperado.

7. Comparación de los niveles teóricos esperados de señal con los obtenidos de manera empírica.

Potencia teórica y real: Como fue mostrado anteriormente en este documento, mediante el MEnUF se obtienen las pérdidas por propagación $\left(\mathrm{L}_{\mathrm{TR}}\right)$ a base de los modelos de Okumura, Hata y Hata-Extendido. Gracias a ello y a los datos entregados en la tabla 2 podemos calcular la potencia de recepción teórica $\left(\mathrm{P}_{\mathrm{RX}}\right)$ de cada uno de los enlaces y así realizar la comparación con los resultados obtenidos $\left(\mathrm{P}_{\mathrm{RX}}\right.$ real) en las mediciones realizadas.

En la tabla 2 podemos observar, por columnas, la distancia $(\mathrm{d},[\mathrm{m}])$ existente entre transmisor y receptor, la sumatoria de ganancias y pérdidas, tanto en el transmisor como en el receptor, los valores experimentales de la potencia de señal recibida (Prx) $[\mathrm{dBm}]$ y los valores teóricos de la potencia de recepción al aplicar los modelos de Propagación en Espacio Libre (E.L.), Okumura (Oku), Hata (H) y Hata-Extendido (HE). Luego se tiene las columnas en que se indica el error (E) obtenido al comparar cada una de las mediciones con los modelos de predicción. Éstos, en promedio, superan el $10 \%$. Esto se explica por el hecho de que los modelos consideran terminales móviles, según lo señalado. En la misma tabla 2 podemos observar a continuación el grado de obstrucción según el MEnUF (Obst.).

Como fue explicado anteriormente, el MEnUF toma como base los modelos de Okumura, Hata y HataExtendido, los cuales son modificados por medio de las ecuaciones (5) hasta (11), por lo tanto, en la tabla 4.1 también es posible observar las potencias obtenidas al aplicar el MEnUF ( $\left.\mathrm{P}_{\mathrm{RX} \text { Oku }}, \mathrm{P}_{\mathrm{RX} H}, \mathrm{P}_{\mathrm{RX} \mathrm{H}-\mathrm{E}}\right)$. Es posible observar a continuación el error (E) porcentual y el error promedio obtenido por cada uno de los modelos de predicción en la tabla 2.

Finalmente, se observa el margen de desvanecimiento de señal del sistema existente (MDesv), el cual indica la diferencia entre la potencia recibida medida y el nivel mínimo de señal requerido por el receptor y, lo más importante, indica el grado de robustez de cada uno de los enlaces, ya que mientras mayor sea el enlace será menos susceptible a errores en la recepción de los datos. Para que un enlace sea considerado como robusto, se recomienda que el margen de desvanecimiento de la señal sea alrededor de $20 \mathrm{~dB}$ (o al menos superior a $10 \mathrm{~dB}$ ), considerando que se trata de enlaces fijos, pero sometidos a variaciones debido a cambios en el entorno.

De la tabla 2 se desprende que el modelo que mejor predice la potencia de recepción, de acuerdo al grado de obstrucción es el modelo MEnUF basado en el modelo de Okumura (en negrita), ya que este modelo es el que tiene un menor error promedio y la menor desviación, lo que implica que el grado de predicción es mejor. También en esta tabla es posible apreciar que existen dos mediciones ( $\mathrm{N}^{\circ} 4$ y $\left.\mathrm{N}^{\circ} 13\right)$ en las cuales el nivel esperado de la señal, según el MEnUF, presenta un error fuera de lo común. Los dos casos tienen una explicación. En la primera medición, el sector donde se ubica el receptor posee el mayor nivel de tránsito vehicular en la ciudad de Valparaíso; por lo tanto, debido a lo anterior se produce el efecto de multitrayectorias erráticas que implican grandes variaciones en la señal recibida, lo que agrega una atenuación extra al enlace. En la segunda medición, el error excesivo se debe a dos factores, la presencia de un edificio de gran altura, el cual obstruye de gran manera la línea de visión directa del enlace lo que agrega un efecto filo de cuchillo. La atenuación debida a este efecto fue agregada a los cálculos, pero no de una manera exacta, lo que explica el error fuera de lo común obtenido. Además, existe la posibilidad de que la antena receptora no estuviera apuntada de una manera óptima y como ésta posee una gran directividad las pérdidas por este efecto son considerables. 
Tabla 2 Potencias obtenidas por los modelos de Okumura, Hata y Hata-Extendido y sus versiones modificadas respecto a las mediciones reales.

\begin{tabular}{|c|c|c|c|c|c|c|c|c|c|c|c|c|c|c|c|c|c|}
\hline \multirow[b]{2}{*}{$\mathbf{N}^{\circ}$} & \multirow[b]{2}{*}{ d [m] } & \multicolumn{5}{|c|}{ Prx [dBm] } & \multicolumn{4}{|c|}{ Error [\%] } & \multirow[t]{2}{*}{ Obstr. } & \multicolumn{3}{|c|}{ Prx MEnUF (dBm) } & \multicolumn{3}{|c|}{ Error [\%] } \\
\hline & & Real & E. $\mathbf{L}$ & Oku & $\mathbf{H}$ & H-E & E. $\mathbf{L}$ & Oku & $\mathbf{H}$ & H-E & & Oku & $\mathbf{H}$ & H-E & Oku & $\mathbf{H}$ & H-E \\
\hline 1 & 1305 & $-68,0$ & $-53,5$ & $-74,9$ & $-68,1$ & $-74,0$ & 27,2 & 9,2 & 0,1 & 8,1 & $\mathrm{C}$ & $-66,3$ & $-62,2$ & $-65,8$ & 2,52 & 9,25 & 3,37 \\
\hline 2 & 1398 & $-60,5$ & $-56,1$ & $-76,4$ & $-58,9$ & $-64,8$ & 7,8 & 20,8 & 2,7 & 6,7 & $P$ & $-62,2$ & $-56,9$ & $-58,7$ & 2,69 & 6,27 & 3,07 \\
\hline 3 & 3500 & $-63,3$ & $-50,3$ & $-65,0$ & $-50,7$ & $-56,6$ & 25,7 & 2,7 & 24,7 & 11,7 & $\mathrm{G}$ & $-63,5$ & $-50,7$ & $-56,0$ & 0,44 & 24,82 & 12,96 \\
\hline 4 & 1545 & $-66,0$ & $-53,6$ & $-68,6$ & $-61,5$ & $-67,4$ & 23,1 & 3,8 & 7,3 & 2,1 & $\mathrm{P}$ & $-58,1$ & $-56,0$ & $-57,8$ & 13,56 & 17,88 & 14,27 \\
\hline 5 & 4317 & $-70,0$ & $-65,9$ & $-77,0$ & $-63,8$ & $-69,7$ & 6,3 & 9,1 & 9,8 & 0,5 & $\mathrm{C}$ & $-72,5$ & $-67,1$ & $-69,0$ & 3,45 & 4,32 & 1,46 \\
\hline 6 & 492 & $-42,0$ & $-43,7$ & $-46,4$ & $-34,2$ & $-40,1$ & 3,8 & 9,5 & 22,8 & 4,7 & $\mathrm{~L}$ & $-44,0$ & $-44,6$ & $-43,3$ & 4,46 & 5,89 & 3,07 \\
\hline 7 & 477 & $-39,0$ & $-40,1$ & $-45,1$ & $-42,1$ & $-48,1$ & 2,8 & 13,5 & 7,5 & 18,8 & $\mathrm{~L}$ & $-40,6$ & $-40,3$ & $-40,9$ & 3,96 & 3,27 & 4,67 \\
\hline 8 & 234 & $-41,0$ & $-37,2$ & $-40,1$ & $-27,9$ & $-33,8$ & 10,1 & 2,1 & 47,1 & 21,4 & $\mathrm{P}$ & $-38,1$ & $-40,0$ & $-38,3$ & 7,61 & 2,40 & 7,16 \\
\hline 9 & 3390 & $-58,0$ & $-57,2$ & $-80,4$ & $-65,2$ & $-71,1$ & 1,5 & 27,9 & 11,1 & 18,5 & $\mathrm{~L}$ & $-59,5$ & $-58,0$ & $-57,3$ & 2,47 & 0,07 & 1,19 \\
\hline 10 & 3296 & $-60,0$ & $-60,2$ & $-89,3$ & $-75,7$ & $-81,7$ & 0,3 & 32,8 & 20,8 & 26,5 & $\mathrm{~L}$ & $-63,1$ & $-61,7$ & $-62,4$ & 4,93 & 2,77 & 3,77 \\
\hline 11 & 1575 & $-58,0$ & $-52,0$ & $-59,4$ & $-45,5$ & $-51,4$ & 11,6 & 2,3 & 27,4 & 12,8 & $P$ & $-54,2$ & $-53,9$ & $-52,2$ & 7,01 & 7,55 & 11,22 \\
\hline 12 & 1535 & $-54,0$ & $-53,6$ & $-61,0$ & $-47,0$ & $-52,9$ & 0,8 & 11,5 & 14,8 & 2,0 & $P$ & $-55,8$ & $-55,5$ & $-53,8$ & 3,24 & 2,76 & 0,45 \\
\hline 13 & 789 & $-74,0$ & $-54,4$ & $-56,9$ & $-44,8$ & $-50,7$ & 36,1 & 30,1 & 65,1 & 45,8 & $\mathrm{G}$ & $-56,6$ & $-63,0$ & $-57,7$ & 30,70 & 17,48 & 28,32 \\
\hline 14 & 1093 & $-62,0$ & $-57,2$ & $-63,2$ & $-49,0$ & $-54,9$ & 8,4 & 1,9 & 26,6 & 13,0 & $\mathrm{P}$ & $-59,0$ & $-59,7$ & $-57,9$ & 5,07 & 3,87 & 7,06 \\
\hline 15 & 1856 & $-58,0$ & $-55,2$ & $-62,5$ & $-48,7$ & $-54,6$ & 5,0 & 7,2 & 19,1 & 6,2 & $\mathrm{~L}$ & $-55,9$ & $-55,9$ & $-55,3$ & 3,68 & 3,81 & 4,92 \\
\hline 16 & 1147 & $-61,0$ & $-57,6$ & $-63,5$ & $-52,6$ & $-58,5$ & 5,8 & 3,9 & 16,0 & 4,3 & $\mathrm{P}$ & $-59,4$ & $-59,2$ & $-57,9$ & 2,73 & 3,13 & 5,35 \\
\hline 17 & 1880 & $-61,6$ & $-52,0$ & $-78,0$ & $-54,8$ & $-60,7$ & 18,4 & 21,1 & 12,4 & 1,5 & $\mathrm{P}$ & $-59,9$ & $-52,9$ & $-54,6$ & 2,82 & 16,52 & 12,74 \\
\hline 18 & 4877 & $-62,1$ & $-60,3$ & $-81,7$ & $-62,7$ & $-68,6$ & 3,0 & 23,9 & 0,9 & 9,4 & $P$ & $-66,7$ & $-61,0$ & $-62,8$ & 6,87 & 1,82 & 1,07 \\
\hline 19 & 4877 & $-62,5$ & $-60,3$ & $-81,7$ & $-62,7$ & $-68,6$ & 3,6 & 23,5 & 0,4 & 9,0 & $\mathrm{P}$ & $-66,7$ & $-61,0$ & $-62,8$ & 6,37 & 2,36 & 0,54 \\
\hline 20 & 2085 & $-60,7$ & $-52,9$ & $-78,8$ & $-72,3$ & $-78,3$ & 14,7 & 22,9 & 16,1 & 22,4 & $\mathrm{P}$ & $-60,7$ & $-58,8$ & $-60,5$ & 0,03 & 3,32 & 0,28 \\
\hline 21 & 4212 & $-61,0$ & $-59,0$ & $-81,0$ & $-74,3$ & $-80,2$ & 3,2 & 24,8 & 18,0 & 24,0 & $\mathrm{P}$ & $-65,8$ & $-63,6$ & $-65,4$ & 7,38 & 4,18 & 6,78 \\
\hline 22 & 2154 & $-54,5$ & $-53,2$ & $-69,6$ & $-65,2$ & $-71,2$ & 2,4 & 21,7 & 16,4 & 23,4 & $\mathrm{~L}$ & $-54,9$ & $-54,4$ & $-55,0$ & 0,62 & 0,18 & 0,89 \\
\hline 23 & 503 & $-49,7$ & $-40,6$ & $-50,3$ & $-47,9$ & $-53,8$ & 22,4 & 1,3 & 3,7 & 7,7 & $\mathrm{C}$ & $-46,4$ & $-45,0$ & $-48,5$ & 7,00 & 10,45 & 2,37 \\
\hline 24 & 4856 & $-62,0$ & $-60,3$ & $-86,7$ & $-67,3$ & $-73,2$ & 2,9 & 28,4 & 7,7 & 15,2 & $\mathrm{P}$ & $-68,2$ & $-62,2$ & $-64,1$ & 9,02 & 0,24 & 3,27 \\
\hline \multicolumn{7}{|c|}{ Error Promedio } & 10,3 & 14,8 & 16,6 & 13,2 & & \multicolumn{3}{|c|}{ Error Promedio } & $\mathbf{5 , 7 7}$ & 6,44 & 5,84 \\
\hline & & & & & & & & & & & & \multicolumn{3}{|c|}{ Desv. Est. } & & 6,44 & 5,84 \\
\hline
\end{tabular}

Las mediciones 18 y 19 fueron realizadas sin mover la antena receptora. La diferencia está en que la primera fue realizada a medianoche, donde el tráfico vehicular fue casi nulo y la segunda fue realizada al mediodía, donde existía un tráfico vehicular considerado como bajo. Se puede apreciar que la diferencia de potencia de recepción es mínima $(0,4 \mathrm{~dB})$. La diferencia existente entre estos enlaces y el enlace número cuatro consiste en el flujo vehicular, siendo de aproximadamente 1 vehículo cada 2 segundos en la última; en cambio, en el enlace 19 el flujo vehicular es de aproximadamente 1 móvil cada 3 minutos.

\section{TIEMPO DE COHERENCIA}

El tiempo de coherencia es un parámetro estadístico, utilizado en el diseño de los ecualizadores de canal, ya que determinan el tiempo en que el ajuste de sus parámetros de recepción a base de una secuencia de entrenamiento para adecuar su respuesta al canal es válido [2]. La forma de obtener el tiempo de coherencia es por medio de la autocovarianza de la señal recibida, y se estima que el tiempo de coherencia del canal está dado por el intervalo de tiempo en que esta función se mantiene sobre el $50 \%$ de su valor máximo [2].

La autocorrelación de un proceso $x(t)$, real o complejo, es por definición el promedio estadístico o esperanza del producto $x\left(t_{1}\right) x^{*}\left(t_{2}\right)$. Esta función se denota $R_{x x}\left(t_{p} t_{2}\right)$. Entonces, $R_{x x}\left(t_{p}, t_{2}\right)=E\left\{x\left(t_{1}\right) x^{*}\left(t_{2}\right)\right\}$ donde, el término conjugado $\left(x^{*}(\cdot)\right)$ es asociado a la segunda variable en $R_{x x}\left(t_{p}, t_{2}\right)$. De lo anterior se desprende la ecuación (12):

$$
R_{x x}\left(t_{2}, t_{1}\right)=E\left\{x\left(t_{2}\right) x *\left(t_{1}\right)\right\}=R_{x x}^{*}\left(t_{1}, t_{2}\right)
$$

La autocovarianza $C_{x}\left(t_{p}, t_{2}\right)$ de un proceso $x(t)$ es la covarianza de las variables aleatorias $x\left(t_{p}\right)$ y $x\left(t_{2}\right)$ :

$$
C_{x}\left(t_{1}, t_{2}\right)=R_{x}\left(t_{1}, t_{2}\right)-\eta\left(t_{1}\right) \eta *\left(t_{2}\right)
$$

donde, $h(t)=E[x(t)]$, el valor medio de $\mathrm{x}(\mathrm{t})$.

Observación: La autocovarianza $C_{x}\left(t_{p}, t_{2}\right)$ de un proceso $x(t)$ es la autocorrelación de un proceso centrado $x(t)=x(t)-h(t)$. 
Los tiempos de coherencia mostrados en la tabla 3 corresponden a medidas obtenidas con transmisiones en banda ancha $(11 \mathrm{Mb} / \mathrm{s})[3]$. Para fines de identificación del enlace se señala en clave la ubicación del transmisor y del receptor, que corresponden a los colegios en que se realizó la medición. Es interesante notar que un paquete de unos 1.500 bytes (tamaño máximo de un paquete Ethernet) se transmite en un tiempo menor que el tiempo de coherencia del canal, para tasas de transmisión entre $1 \mathrm{Mb} / \mathrm{s}$ y $11 \mathrm{Mb} / \mathrm{s}$, lo que significa que el canal presenta características que permiten aprovechar el entrenamiento de los ecualizadores mientras se reciben los paquetes de datos usando este protocolo. Estas medidas son coherentes con otras medidas establecidas con técnicas de medición de banda estrecha, que se han estado realizando paralelamente en el Departamento sobre otros enlaces en la misma banda y que están en vías de ser publicados.

Tabla 3 Tiempo de coherencia de canal de algunos enlaces medidos.

\begin{tabular}{|c|c|c|c|c|}
\hline \multicolumn{5}{|c|}{ Tiempo de Coherencia de Canal } \\
\hline $\mathrm{N}^{\circ}$ & $\begin{array}{c}\text { Ubicación } \\
\text { Txr }\end{array}$ & Ubicación Receptor & $\mathrm{d}[\mathrm{m}]$ & $\begin{array}{c}\text { Tiempo } \\
\text { coherencia } \\
{[\mathrm{seg} .]}\end{array}$ \\
\hline 1 & UTFSM & Latorre 297, Recreo & 1398 & 0,262 \\
\hline 2 & UTFSM & Vives 630, Puerto & 3500 & 0,223 \\
\hline 3 & Murialdo & Cood 300, Rocuant & 1880 & 0,233 \\
\hline 4 & Murialdo & Chaparro 638, Cord. & 4877 & 0,427 \\
\hline 5 & Murialdo & Chaparro 638, Cord. & 4877 & 0,261 \\
\hline 6 & Murialdo & Veas 19, Ramaditas & 2085 & 0,256 \\
\hline 7 & Murialdo & Márquez 243,Puerto & 4212 & 0,568 \\
\hline 8 & Murialdo & Almte. Latorre 79 & 2151 & 0,273 \\
\hline 9 & Murialdo & Valpso. 129, Barón & 503 & 0,256 \\
\hline 10 & Murialdo & Millalongo 23, Cord & 4856 & 0,278 \\
\hline
\end{tabular}

\section{FACTOR K}

En canales de radiofrecuencias se producen desvanecimientos temporales, que producen fluctuaciones en la amplitud de la señal recibida. Si el ancho de banda de la señal transmitida es menor al ancho de banda de coherencia, las características espectrales de la señal original no presentan grandes alteraciones en el receptor, dando origen a un desvanecimiento plano en frecuencia [2]. Si el canal de radiofrecuencia presenta esta característica, la envolvente de la señal recibida puede ser descrita en función de la distribución estadística de Rice [2].

El Factor $K$ es el parámetro utilizado para describir la distribución de Rice, por lo que es también conocido como Factor de Rice. Este factor esta definido como la razón entre la potencia de la señal y la varianza de la componente multitrayecto (ecuación 14).

$$
K=\frac{A^{2}}{2 \sigma^{2}}
$$

donde:

A : Amplitud de la envolvente de la señal dominante.

$\sigma$ : Valor rms de la señal recibida.

La función de densidad de probabilidad (pdf) de la envolvente de la señal recibida $R(t)$ de acuerdo a la distribución estadística Rice en términos del Factor $\mathrm{K}$ [2] esta dado por las ecuaciones 15 y 16.

$$
p(r)=\frac{2(K+1) r}{\Omega} \exp \left(-K-\frac{(K+1) r^{2}}{\Omega}\right) I_{0}\left(2 r \sqrt{\frac{K(K+1)}{\Omega}}\right)
$$

$r \geq 0, K \geq 0, \Omega \geq 0$

$$
\Omega=E\left[R^{2}\right]=A^{2}+2 \sigma^{2}
$$

Donde:

$\mathrm{r}$ : Envolvente de la señal transmitida.

$\mathrm{I}_{0}$ : Función de Bessel modificada de primer tipo y orden cero.

En este trabajo determinaremos el Factor K mediante el Método de Dos Momentos, el cual es explicado en detalle en [4] y [5].

Se analizaron los datos obtenidos divididos en doce periodos de aproximadamente cinco minutos cada uno para ver la consistencia de las mediciones. En la tabla 4 se presentan los valores promedio y la varianza de $\mathrm{K}$ correspondientes a estos intervalos para cada uno de los enlaces registrados en la tabla 3, como también el valor de este parámetro para la medición completa de una hora (KTotal).

Tabla 4 Factor K para mediciones de cinco minutos y para la medición completa.

\begin{tabular}{|c|l|c|c|c|c|}
\hline $\mathrm{N}^{\circ}$ & $\begin{array}{c}\text { Ubicación } \\
\text { Receptor }\end{array}$ & $\begin{array}{c}\mathrm{d} \\
{[\mathrm{m}]}\end{array}$ & $\begin{array}{c}\text { Factor K } \\
\text { Promedio }\end{array}$ & $\begin{array}{c}\text { Desviación } \\
\text { Factor K }\end{array}$ & $\begin{array}{c}\text { Factor K } \\
1 \text { hora }\end{array}$ \\
\hline 1 & Latorre 297 & 1398 & 140.54 & 12.11 & 136.17 \\
\hline 2 & Vives 630 & 3500 & 6.58 & 1.48 & 6.25 \\
\hline 3 & Cood 300 & 1880 & 90.28 & 27.11 & 83.93 \\
\hline 4 & Chap. 638 & 4877 & 124.22 & 55.60 & 77.83 \\
\hline 5 & Chap. 638 & 4877 & 144.56 & 14.59 & 140.90 \\
\hline 6 & Veas 19 & 2085 & 71.20 & 10.44 & 69.00 \\
\hline 7 & Márq. 243 & 4212 & 60.36 & 9.60 & 39.53 \\
\hline 8 & A. Latorre 79 & 2151 & 133.55 & 38.91 & 115.23 \\
\hline 9 & Valpso. 129 & 503 & 154.63 & 12.62 & 152,22 \\
\hline 10 & Millal. 23 & 4856 & 77.76 & 29.17 & 65.48 \\
\hline
\end{tabular}


Los valores para el Factor $K$ varían entre 6 y 140 aproximadamente y no existe mayor diferencia entre ellos, cuando se recorren por fila. Esto señala que estamos ante un proceso estacionario $\mathrm{y}$, aun más importante, que basta con tomar muestras de un intervalo de 5 minutos. Los casos extremos son los denominados como 2 y 5 . Para el primero caso, se obtiene un Factor K en torno a 6.25 , lo que se debe a que el lugar geográfico donde fue instalado el receptor posee una característica de encajonamiento de la señal, lo que provoca un gran número de señales interferentes. El segundo caso posee un Factor K de 140 aproximadamente, lo que se debe a que el enlace entre transmisor y receptor está muy despejado, por lo que la existencia de multitrayectorias es casi nula.

\section{CONCLUSIONES}

Se ha propuesto en este trabajo un procedimiento simple y confiable de predecir los niveles de potencia recibidos para enlaces fijos de largo alcance para aplicaciones de WiFi, para lo cual es necesario tener presente las clasificaciones definidas para el grado de obstrucción presente en el enlace. Se ha establecido que el modelo predice la calidad del enlace con un margen de error inferior al $10 \%$ en aproximadamente un $87 \%$ de los casos estudiados, mediante visitas inspectivas de una persona medianamente entrenada al sitio donde se desea realizar la instalación de un cliente.

Otro aspecto importante que queda de manifiesto gracias a las mediciones realizadas sobre estos enlaces es que los tiempos de coherencia de canal obtenidos son tales que la transmisión de los datos es posible. Esto se deprende del hecho de que el tiempo de coherencia de cada uno de los enlaces estudiados es mayor al tiempo necesario para trasmitir los paquetes de datos de mayor tamaño que se pueden presentar en una red Ethermet, no importando si la tasa de transmisión del enlace es de 10 $11 \mathrm{Mb} / \mathrm{s}$.

Mientras mayor es el Factor $K$, mejor es el enlace entablado, ya que este parámetro indica la relación existente entre la señal portadora y las señales interferentes en un enlace. Para efectos prácticos, se considera como un valor del Factor $K$ aceptable a aquel que está por sobre 10. Dado que los enlaces medidos fueron diseñados con el criterio de tener al menos línea de vista, se obtienen valores del Factor $K$ relativamente altos y estables.

\section{AGRADECIMIENTOS}

Este trabajo ha sido posible gracias a aportes parciales del proyecto FDI 01 "Difusión Multimedial Inalámbrica IP" y del proyecto UTFSM 230322 "Modelado Estadístico de Canales Inalámbricos en las Bandas de 2.4 y $3.5 \mathrm{GHz}$ ".

\section{REFERENCIAS}

[1] R. Godoy y M. Orellana. "Desarrollo de una red inalámbrica IP en Viña del Mar y Valparaíso". Memoria para optar al título de Ingeniero Civil Electrónico. Departamento de Electrónica. Universidad Técnica Federico Santa María. Valparaíso, Chile. 2003.

[2] T. Rappaport. "Wireless Comunications". $2^{\text {nd }}$ Ed. Prentice Hall. 2002.

[3] Institute of Electrical and Electronics Engineers. "IEEE 802.11b Standard". IEEE. 1999.

[4] A. Abdi, C. Tepedelenlioglu, M. Kaveh and G. Giannakis. "On the Estimation of the K Parameter for the Rice Fading Distribution". IEEE Communications Letters. Vol. 5. No 3. March 2001.

[5] L.J. Greenstein, D.G. Michelson, V. Erceg. "Moment-Method Estimation of the Ricean K-Factor". IEEE Communications Letters. Vol. 3. No6. June 1999.

[6] C. Correa. "Evaluación de las características de enlace de largo alcance carentes de línea de vista para aplicaciones WLL usando protocolo IEEE 802.11 b". Memoria para optar título de Ingeniero Civil Electrónico. Departamento de Electrónica. Universidad Técnica Federico Santa María. Valparaíso, Chile. 2004. 